\title{
Quantum Hall effect in graphene with interface-induced spin-orbit coupling
}

\author{
Tarik P. Cysne, ${ }^{1}$ Jose H. Garcia, ${ }^{2}$ Alexandre R. Rocha,${ }^{3}$ and Tatiana G. Rappoport ${ }^{1}$ \\ ${ }_{1}^{1}$ Instituto de Física, Universidade Federal do Rio de Janeiro, Caixa Postal 68528, Rio de Janeiro 21941-972, Rio de Janeiro, Brazil \\ ${ }^{2}$ Catalan Institute of Nanoscience and Nanotechnology, CSIC and Barcelona Institute of Science and Technology, \\ Campus UAB, 08193 Barcelona, Spain \\ ${ }^{3}$ Instituto de Física Teórica, Universidade Estadual Paulista, Rua Dr. Bento T. Ferraz, 271, São Paulo, São Paulo 01140-070, Brazil
}

(Received 21 November 2017; revised manuscript received 24 January 2018; published 9 February 2018)

\begin{abstract}
We consider an effective model for graphene with interface-induced spin-orbit coupling and calculate the quantum Hall effect in the low-energy limit. We perform a systematic analysis of the contribution of the different terms of the effective Hamiltonian to the quantum Hall effect (QHE). By analyzing the spin splitting of the quantum Hall states as a function of magnetic field and gate voltage, we obtain different scaling laws that can be used to characterize the spin-orbit coupling in experiments. Furthermore, we employ a real-space quantum transport approach to calculate the quantum Hall conductivity and investigate the robustness of the QHE to disorder introduced by hydrogen impurities. For that purpose, we combine first-principles calculations and a genetic algorithm strategy to obtain a graphene-only Hamiltonian that models the impurity.
\end{abstract}

DOI: 10.1103/PhysRevB.97.085413

\section{INTRODUCTION}

The interaction between a graphene sheet and different substrates has attracted great attention in recent years due to the appearance of interesting effects in the graphene layer [1-6]. This is particularly relevant in the context of van der Waals heterostructures [7], vertical stacks of bidimensional materials that promise to lead to novel electronics devices and applications. One possibility in this direction is the use of graphene-based van der Waals heterostructures for spintronics.

Graphene is a nonmagnetic material with very weak spinorbit coupling (SOC) [8-10] due to the lightness of carbon atoms. Since its discovery, there were several proposals to introduce and control spin-dependent properties in graphene by inducing spin-orbit coupling [11-16]. Recently, there has been significant progress in engineering those properties using the proximity effect, which allows introducing spindependent features while preserving graphene's high electronic mobility. Graphene has been proximity coupled with a magnetic thin layer of YIG for transport [17] and spinpumping [18] measurements. SOC was also induced by the proximity effect in graphene on top of different transitionmetal dichalcogenides (TMDCs) [19-22] and graphene decorated with gold [23]. More recently, spins were optically injected in graphene/TMDC systems [24,25], also indicating the presence of SOC in the graphene layer. However, none of these measurements give clear indications of the type of underlying spin-orbit-coupling mechanism that generates the observed phenomena.

In this paper, we exploit the possibility of using quantum Hall measurements to extract the characteristics of the spinorbit coupling in graphene. For that purpose, we employ the effective model for graphene with interface-induced spin-orbit coupling from Ref. [1]. We then use Landau operators to calculate the quantum Hall effect in the low-energy limit. By performing a systematic analysis of the spin splitting of the
Landau levels (LLs) in the quantum Hall regime as a function of magnetic field and gate voltage, we obtain characteristic scaling laws for the splittings produced by each type of spin-orbit coupling. The same type of analysis can be used to characterize and estimate the SOC in transport experiments. Furthermore, we employ a real-space quantum transport approach based on a Chebyshev polynomial expansion of disordered Green's functions to calculate the quantum Hall conductivity [26]. We use this numerical approach to investigate the robustness of the quantum Hall effect (QHE) to disorder introduced by hydrogen impurities. The impurities are modeled by an $a b$ initio-derived tight-binding model. For the extraction of the tight-binding parameters, it is necessary to perform a multiparametric fit. Deterministic approaches for the fit can be quite difficult because of the occurrence of a large number of extrema. Therefore, here, we propose the use of a heuristic algorithm to perform this task efficiently.

This paper is organized as follows: in Sec. II, we introduce the tight-binding and low-energy models for graphene with interface-induced SOC. We also present the Hamiltonian of the system under an external magnetic field, written in terms of Landau operators. In Sec. III, we discuss our analytical results for the energy spectra and Hall conductivity and introduce the scaling laws that can be used to discriminate the different SOC. In Sec. IV we present the heuristic algorithm that was used to extract the tight-binding parameters from density functional theory spectra and our numerical approach for the conductivity calculations. We then discuss the results for the effect of hydrogenation on the QHE for graphene with interface-induced SOC. Finally, we present our conclusions in Sec. V.

\section{THEORY}

With a combination of density functional theory (DFT) and group theory analysis, Gmitra et al. proposed a tight-binding 
Hamiltonian and its corresponding low-energy approximation for graphene stacked on TMDCs [1]. Here, we consider their Hamiltonian with the two most relevant spin-orbit terms for the electronic properties of graphene in the vicinity of the Dirac point:

$$
\begin{aligned}
\mathcal{H}= & \sum_{\langle i, j\rangle} t c_{i s}^{\dagger} c_{j s}+\sum_{i} \Delta \eta_{c_{i}} c_{i s}^{\dagger} c_{i s}+\frac{2 i}{3} \lambda_{\mathrm{R}} \sum_{\langle i, j\rangle} c_{i s}^{\dagger} c_{j s^{\prime}} \\
& \times\left[\left(\hat{\mathbf{s}} \times \mathbf{d}_{i j}\right)_{z}\right]_{s s^{\prime}}+\frac{i}{3} \sum_{\langle\langle i, j\rangle\rangle} \frac{\lambda_{c_{i}}}{\sqrt{3}} c_{i s}^{\dagger} c_{j s^{\prime}} v_{i j} \hat{s}_{z},
\end{aligned}
$$

where $c_{i s}^{\dagger}=\left(a_{i s}^{\dagger}, b_{i s}^{\dagger}\right)$ and $c_{i s}=\left(a_{i s}, b_{i s}\right)$ are the creation and annihilation operators for an electron on a lattice site $i$ and spin $s$ belonging to sublattice A or B, respectively. The first term represents the hopping with amplitude $t$ between $\pi$ orbitals in a honeycomb lattice, and the second term is an energy offset $\Delta$ between sublattices $\mathrm{A}\left(\eta_{a_{i}}=1\right)$ and $\mathrm{B}$ $\left(\eta_{b_{i}}=-1\right)$ due to the superlattice effect originated by the incommensurability of the two lattices. The third contribution is the typical Rashba SOC with strength $\lambda_{R}[27,28]$. It arises because the inversion symmetry is broken when graphene is placed on top of a TMDC. The fourth contribution is a sublattice-dependent intrinsic SOC with coupling intensities $\lambda_{a}$ and $\lambda_{b} . H_{\xi}$, the valley-dependent low-energy limit of the Hamiltonian of Eq. (1), has four terms [1],

$$
\begin{aligned}
H_{\xi}= & \hbar v_{f}\left(\xi \sigma_{x} k_{x}+\sigma_{y} k_{y}\right)+\Delta \sigma_{z}+\lambda_{\mathrm{R}}\left(\xi \sigma_{x} s_{y}-\sigma_{y} s_{x}\right) \\
& +\frac{1}{2} \xi\left[\lambda_{a}\left(\sigma_{z}+\sigma_{0}\right)+\lambda_{b}\left(\sigma_{z}-\sigma_{0}\right)\right] s_{z} .
\end{aligned}
$$

The first two terms are the spinless contributions, where $v_{F}$ is the Fermi velocity given by $v_{F}=\frac{3 t a}{2 \hbar}$ with lattice constant $a, \sigma$ is a pseudospin Pauli matrix related to sublattices $\mathrm{A}$ and $\mathrm{B}$, and $k_{x}$ and $k_{y}$ are the components of the electronic moment relative to the Dirac points. $\xi$ is related to the valley degree of freedom; $\xi$ is positive for valley $K$ and negative for valley $K^{\prime}$. The third and fourth terms are the Rashba and sublattice-resolved intrinsic spin-orbit couplings, respectively. The fourth term contains the well-known Kane-Mele term [29,30] with strength $\lambda_{\mathrm{I}}=\left(\lambda_{a}+\lambda_{b}\right) / 2$ and a valley Zeeman SOC with strength $\lambda_{\mathrm{VZ}}=\left(\lambda_{a}-\lambda_{b}\right) / 2$ that couples spin and valley degrees of freedom.

In Fig. 1 we show the energy spectrum of the Hamiltonian of Eq. (2) for different combinations of $\lambda_{a}, \lambda_{b}$, and $\lambda_{R}$ to better understand the characteristics of the novel intrinsic spin-orbit coupling. Different from the usual case of $\lambda_{a}=\lambda_{b}$, if only the valley Zeeman contribution $\lambda_{a}=-\lambda_{b}$ (i.e., $\lambda_{\mathrm{VZ}}=\lambda_{a}$ ) is present, the spectrum is gapless, and the spin degeneracy is broken [see Fig. 1(a)]. If only Rashba SOC is present, the spectrum is also gapless [28]. However, any combination $\lambda_{R} \neq 0$ and $\lambda_{\mathrm{VZ}} \neq 0$ opens a gap in the energy spectrum, as shown in Figs. 1(b) and 1(c). For comparison, Fig. 1(d) presents the energy spectrum for $\lambda_{a}=\lambda_{b}$ (i.e., $\lambda_{\mathrm{I}}=\lambda_{a}$ ) and $\lambda_{R} \neq 0$. It is important to note that valley Zeeman combined with Rashba coupling preserves particle-hole symmetry [Figs. 1(b) and 1(c)], while, when Kane-Mele and Rashba terms are both present, the particle-hole symmetry is broken [Fig. 1(d)].

If a uniform perpendicular magnetic field is applied, $\vec{p} \rightarrow$ $\vec{\pi}=\vec{p}+e \vec{A}$, where $\vec{A}$ is the vector potential in the Landau gauge $\vec{A}=B(-y, 0,0)$ and $B$ is the intensity of the magnetic
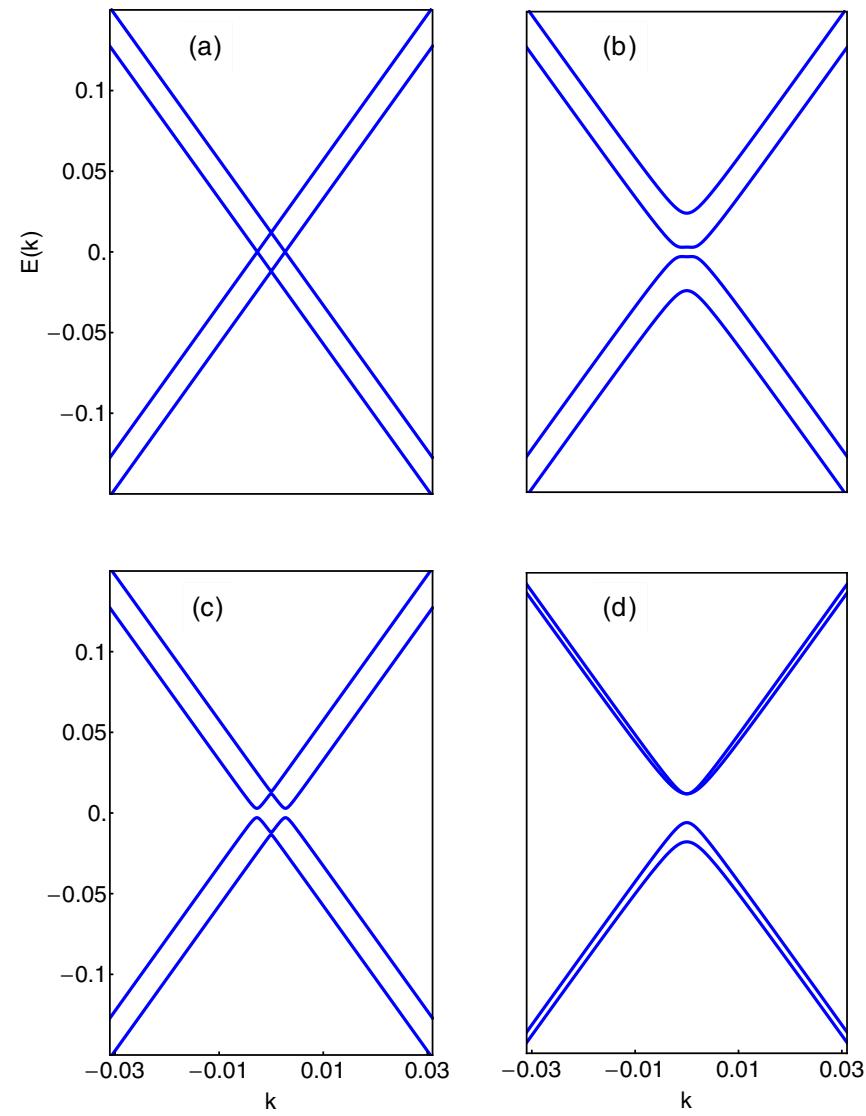

FIG. 1. Energy spectrum of the effective Hamiltonian of Eq. (2) for $\Delta=0$ : (a) $\lambda_{\mathrm{R}}=0$ and $\lambda_{\mathrm{VZ}} \neq 0$, (b) $\lambda_{\mathrm{VZ}} \ll \lambda_{\mathrm{R}}$, (c) $\lambda_{\mathrm{VZ}} \gg \lambda_{\mathrm{R}}$, and (d) $\lambda_{\mathrm{I}} \gg \lambda_{\mathrm{R}}$.

field. The low-energy Hamiltonian can be written as

$$
\begin{aligned}
H_{\xi}= & \hbar \omega\left(\sigma_{\xi} a_{\xi}+\sigma_{-\xi} a_{\xi}^{\dagger}\right)-2 i \xi \lambda_{\mathrm{R}}\left(\sigma_{-\xi} s_{+}-\sigma_{\xi} s_{-}\right) \\
& +\frac{1}{2} \xi\left[\lambda_{a}\left(\sigma_{z}+\sigma_{0}\right)+\lambda_{b}\left(\sigma_{z}-\sigma_{0}\right)\right] s_{z}+\Delta \sigma_{z}
\end{aligned}
$$

where $\sigma_{ \pm}=(1 / 2)\left(\sigma_{x} \pm i \sigma_{y}\right), s_{ \pm}=(1 / 2)\left(s_{x} \pm i s_{y}\right)$, the cyclotron frequency is given by $\omega=\sqrt{2} v_{F} / l_{B}$, and the magnetic length is $l_{B}=\sqrt{\hbar / e B}$. Landau operators $a_{\xi}$ are creation and annihilation operators on valleys $K$ and $K^{\prime}$ for $\xi= \pm$ :

$$
\begin{aligned}
& a_{\xi}=\frac{1}{\sqrt{2}} \xi\left(\frac{l_{B}}{\hbar} p_{x}-\frac{1}{l_{B}} y+\frac{l_{B}}{i \hbar} p_{y}\right)_{\xi}, \\
& a_{\xi}^{\dagger}=\frac{1}{\sqrt{2}} \xi\left(\frac{l_{B}}{\hbar} p_{x}-\frac{1}{l_{B}} y-\frac{l_{B}}{i \hbar} p_{y}\right)_{\xi} .
\end{aligned}
$$

The Hamiltonians $H_{\xi= \pm}$ are block diagonal, with each block indexed by an occupation number $n$. The two lowest blocks are $1 \times 1$ and $3 \times 3$ matrices, and higher blocks are $4 \times 4$ matrices for both valleys. The energies $E_{n, i}^{\xi}$ and eigenvectors $\left|\psi_{n, i}^{\xi}\right\rangle$ are indexed by the valley $\xi$, the occupation number $n$, and $i$, which labels the eigenvalues and eigenvectors of a given block $n$. For more details, see the Appendix. The transverse Hall conductivity $\sigma_{x y}$ can be calculated in the framework of 

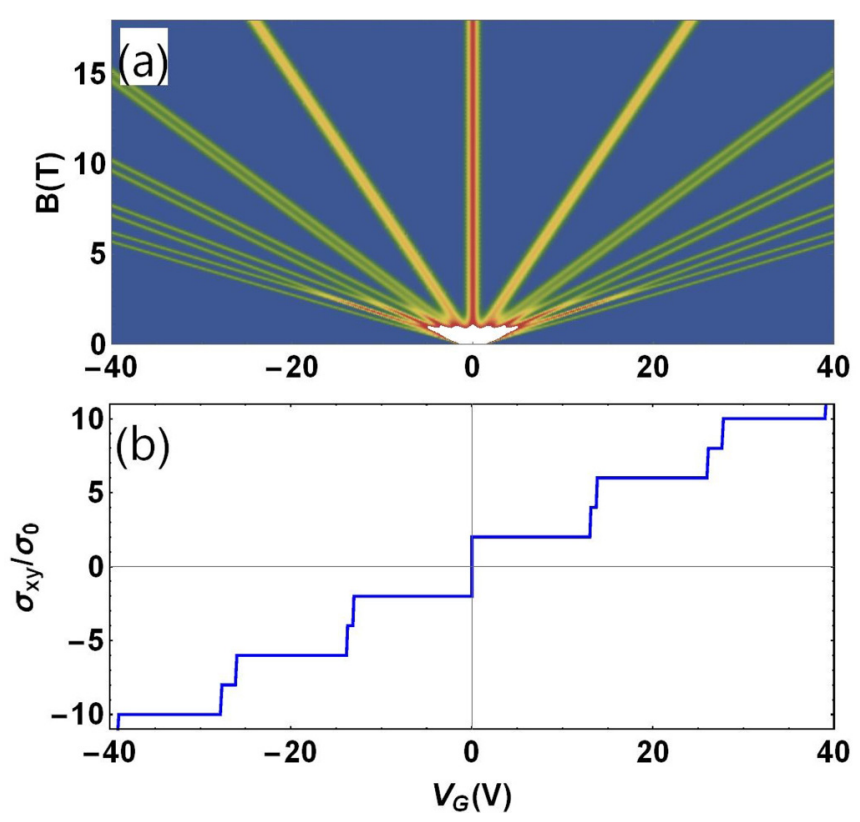

FIG. 2. (a) Landau fan diagram and (b) Hall conductivity as a function of gate voltage for $\lambda_{\mathrm{R}}=10 \mathrm{meV}, \lambda_{\mathrm{VZ}}=0$, and $B=10 \mathrm{~T}$.

the Kubo formula [31]

$$
\begin{aligned}
\sigma_{x y}= & \frac{i e^{3} B v_{F}^{2}}{2 \pi} \sum_{\xi= \pm} \sum_{n, n^{\prime}} \sum_{i, i^{\prime}}\left[f\left(E_{n, i}^{\xi}\right)-f\left(E_{n^{\prime}, i^{\prime}}^{\xi}\right)\right] \\
& \times \frac{\left\langle\psi_{n, i}^{\xi}\left|\sigma_{x}\right| \psi_{n^{\prime}, i^{\prime}}^{\xi}\right\rangle\left\langle\psi_{n^{\prime}, i^{\prime}}^{\xi}\left|\sigma_{y}\right| \psi_{n, i}^{\xi}\right\rangle}{\left(E_{n, i}^{\xi}-E_{n^{\prime}, i^{\prime}}^{\xi}\right)\left(E_{n, i}^{\xi}-E_{n^{\prime}, i^{\prime}}^{\xi}+i 0^{+}\right)},
\end{aligned}
$$

where $f(E)$ is the Fermi-Dirac distribution function. We will not consider thermal effects here, and the Fermi-Dirac distribution has a Heaviside function profile, $f(E)=\Theta\left(E_{f}-\right.$ $E)$. Expressions for the eigenvectors are computed in the Appendix.

\section{LOW-ENERGY CALCULATIONS}

\section{A. Weak spin-orbit coupling}

Here, we show and discuss our results on the quantum Hall effect under the effect of Rashba and valley Zeeman SOCs, which are present in graphene-TMDC heterostructures. Experiments and first-principles calculations point to values of the coupling constants in the range of $0.1-10 \mathrm{meV}[21,22]$. We express our results in terms of a gate voltage which controls the Fermi energy. This voltage is related to the electrons' Fermi momentum in graphene by $k_{f}=E_{f} / \hbar v_{f}=\sqrt{\alpha \pi V_{g}}$ [32], where $\alpha$ depends on the substrate. Here, we use $\alpha=$ $7.2 \times 10^{10} \mathrm{~V}^{-1} \mathrm{~cm}^{-2}$, which is an appropriate value for either silicon oxide or TMDC substrates.

To be compatible with the analysis of experimental results, instead of showing our energy spectra, we present fan diagrams of the density of states as a function of gate voltage $V_{G}$ and magnetic field $B$. We begin by presenting the well-known spin splitting generated by $\lambda_{R}$. In this case, the $n=0$ Landau level is spin degenerate, while all other levels are spin split, as illustrated in Fig. 2. It is also clear from the Landau level splittings of Fig. 2(a) and the quantum Hall conductivity of
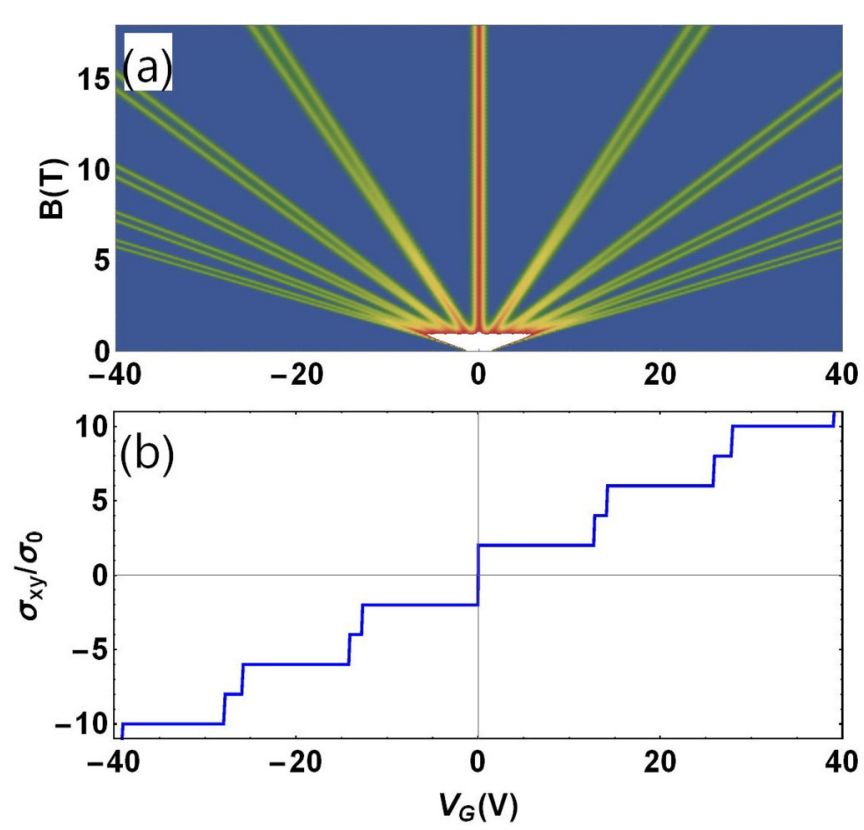

FIG. 3. (a) Landau fan diagram and (b) Hall conductivity as a function of gate voltage for $\lambda_{\mathrm{R}}=0, \lambda_{\mathrm{VZ}}=3 \mathrm{meV}$, and $B=10 \mathrm{~T}$.

Fig. 2(b) that, as expected, the splitting increases with the level number $n$.

We proceed to analyze the spin-splitting generated by $\lambda_{\mathrm{VZ}}$ : like in the previous case, the $n=0$ Landau is spin degenerate, while all other levels are spin split, as illustrated in Fig. 3. Again, the Landau level splittings in Fig. 3(a) and the quantum Hall conductivity in Fig. 3(b) show the increase of the splitting with the level number $n$, and it is considerably larger than the spin splitting produced by Rashba SOC, even for weak couplings and small $n$.

To compare the spin splitting produced by Rashba SOC with the one produced by the valley Zeeman SOC, we select the difference in energy of the $n=3$ levels $\Delta V_{G}$ as a function of the spin-orbit strength for various values of the external magnetic field. From Fig. 4, we see a very different dependence of $\Delta V_{G}$ as a function of $\lambda_{\mathrm{R}}$ [Fig. 4(a)] and $\lambda_{\mathrm{VZ}}$ [Fig. 4(b)]. $\Delta V_{G}$ varies linearly with $\lambda_{\mathrm{VZ}}$ and quadratically with $\lambda_{\mathrm{R}}$. For the case of valley Zeeman SOC, $\Delta V_{G}$ is $1-5 \mathrm{~V}$ even for very weak couplings of the order of $\lambda_{\mathrm{VZ}}=1-5 \mathrm{meV}$ and can be resolved experimentally.

The combined effect of Rashba and valley Zeeman SOCs in the weak-coupling regime can be seen in Figs. 5 and 6 . In Fig. 5, we consider the case where $\lambda_{\mathrm{VZ}}>\lambda_{\mathrm{R}}$, while in Fig. 6 we consider the case where $\lambda_{\mathrm{VZ}}<\lambda_{\mathrm{R}}$. Because of the different functional laws presented in Fig. 4, if both couplings have similar strengths, the splitting is dominated by $\lambda_{\mathrm{VZ}}$. However, as can be seen in these figures, it is difficult to extract information on the nature and strength of the spin-orbit coupling in a graphene heterostructure from fan diagrams and quantum Hall measurements. The spin splitting of the Landau levels and the quantum Hall plateaus for graphene with valley Zeeman SOC are very similar to the ones for graphene with Rashba SOC.

To address this issue, we found two different scaling laws for the spin-split states that can be used to distinguish between 

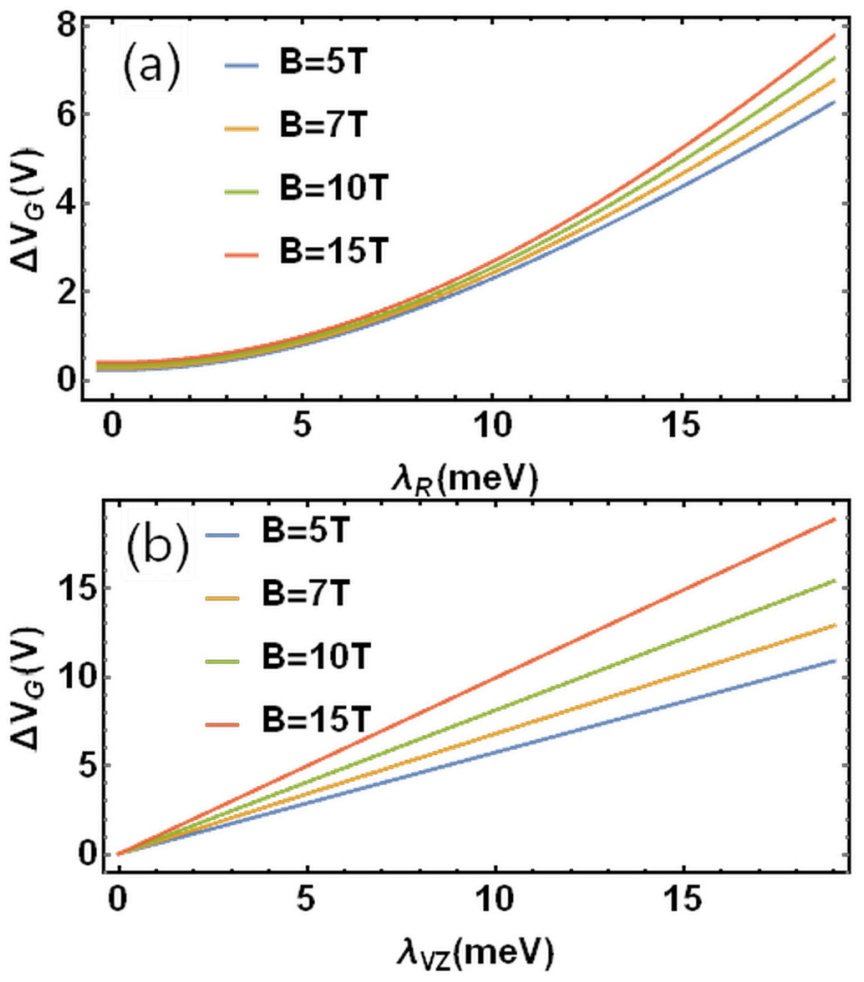

FIG. 4. (a) Splitting $\Delta V_{G}$ of the $n=3$ Landau level as a function of $\lambda_{\mathrm{R}}$ with $\lambda_{\mathrm{VZ}}=0.4 \mathrm{meV}$ fixed and different magnetic fields. (b) Voltage splitting $\Delta V_{G}$ of the $n=3$ Landau level as a function of $\lambda_{\mathrm{VZ}}$ with $\lambda_{\mathrm{R}}=1 \mathrm{meV}$ fixed and different magnetic fields.

systems with valley Zeeman SOC and ones with Rashba SOC. For the case of valley Zeeman SOC, the scaled quantity is $\Delta V_{G}$, the voltage difference between spin-split states with same quantum number $n$. If we consider $\Delta V_{G}^{2} / n$ as a function of the magnetic field, all curves for different values of $n$ collapse
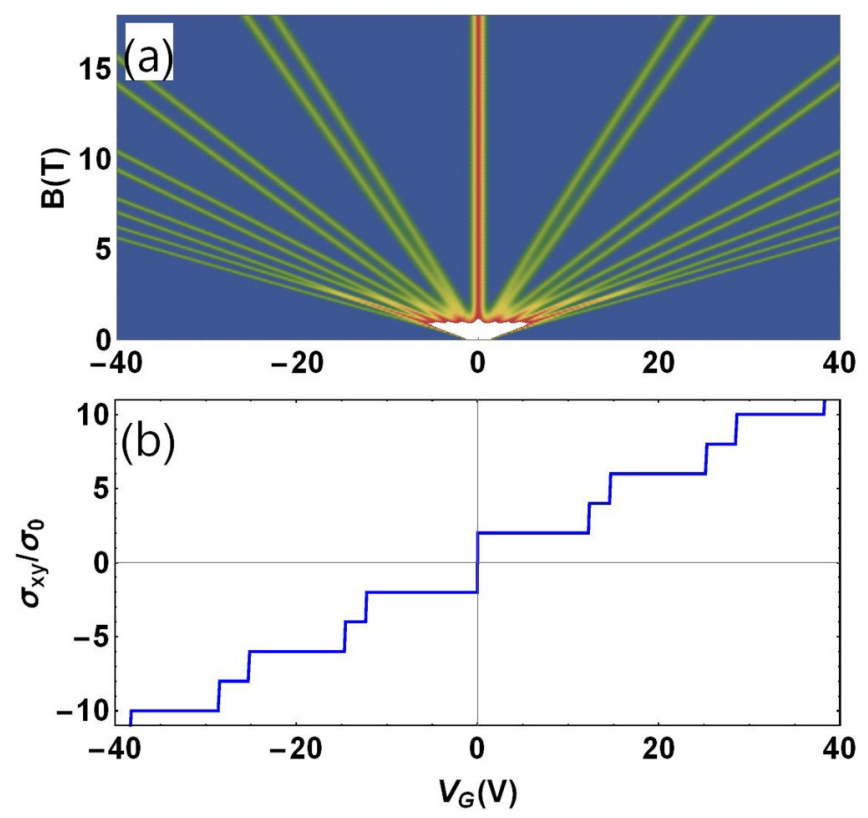

FIG. 5. (a) Landau fan diagram and (b) Hall conductivity as a function of gate voltage for $\lambda_{\mathrm{R}}=1 \mathrm{meV}, \lambda_{\mathrm{VZ}}=5 \mathrm{meV}$, and $B=10 \mathrm{~T}$.

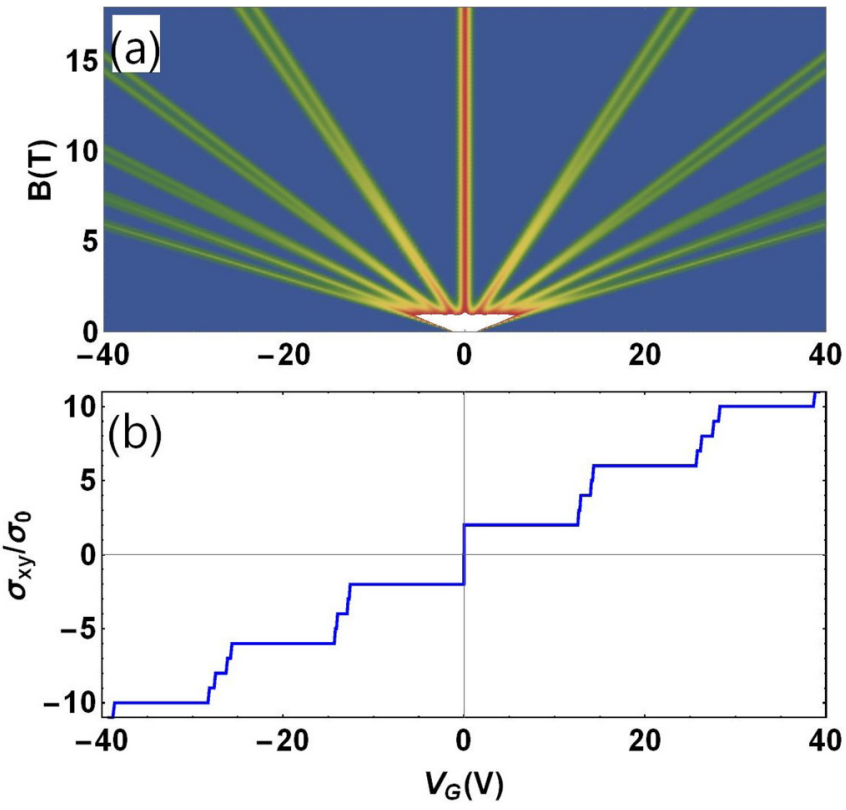

FIG. 6. (a) Landau fan diagram and (b) Hall conductivity as a function of gate voltage for $\lambda_{\mathrm{R}}=6 \mathrm{meV}, \lambda_{\mathrm{VZ}}=3 \mathrm{meV}$, and $B=10 \mathrm{~T}$.

perfectly into a single one, as can be seen in Fig. 7(a). If $\lambda_{R} \neq 0$, the scaling starts to fail whenever the main contribution for the spin splitting is $\lambda_{\mathrm{R}}$ [see Figs. 7(b) and 7(c)], indicating that this scaling law is a characteristic of valley Zeeman SOC.

On the other hand, if we consider $\lambda_{\mathrm{R}} \neq 0$ and $\lambda_{\mathrm{VZ}}=0$, we need to use a different scaling law to collapse all curves. In this case, we use the quantity $4\left(V_{G, s}^{2}-V_{G, s^{\prime}}^{2}\right) / n^{2}$, where $V_{G, s}$ and $V_{G, s^{\prime}}$ are the voltages of the two spin-split states (represented by $s$ and $s^{\prime}$ ) for a given $n$ (see Fig. 8). This simple analysis, which can be used in transport measurements, is able to determine the main SOC in graphene, and the deviations from the scaling laws can also estimate the relative contributions of valley Zeeman and Rashba SOCs.

\section{B. Strong-spin-orbit-coupling regime}

Let us now discuss the case where the spin-orbit coupling is of the order of tens of meV, as estimated via anti-weaklocalization measurements on graphene-TMDC heterostruc-
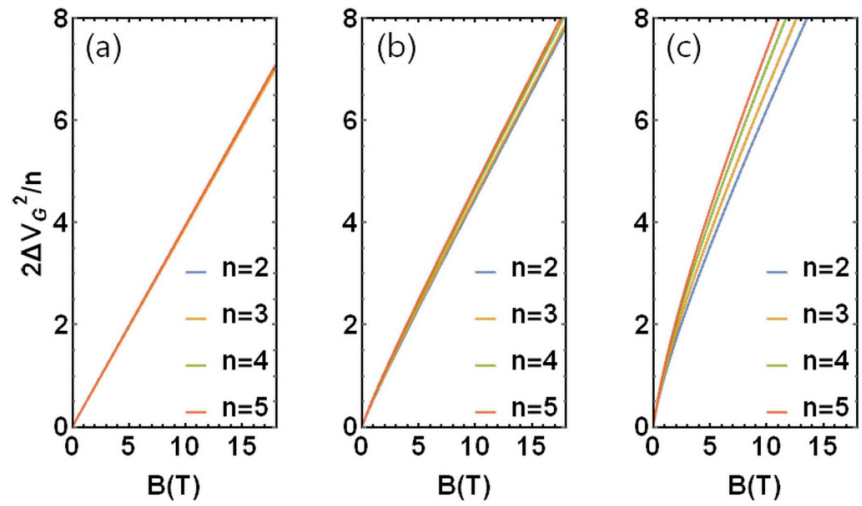

FIG. 7. $2 \Delta V_{G}^{2} / n$ as a function of the magnetic field $B$ for Landau levels $n=2,3,4,5$ with $\lambda_{\mathrm{VZ}}=3 \mathrm{meV}$ and (a) $\lambda_{\mathrm{R}}=0$, (b) $\lambda_{\mathrm{R}}=$ $3 \mathrm{meV}$, and (c) $\lambda_{\mathrm{R}}=6 \mathrm{meV}$. 

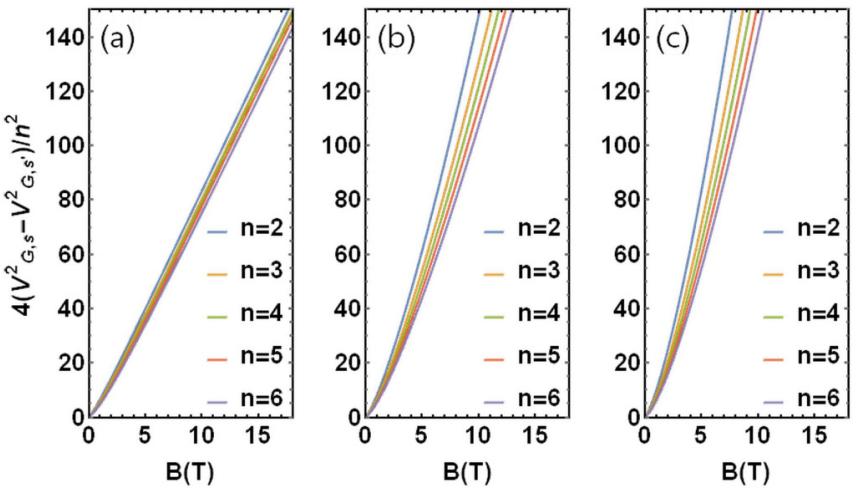

FIG. 8. $4\left(V_{G, s}^{2}-V_{G, s^{\prime}}^{2}\right) / n^{2}$ as a function of the magnetic field $B$ for Landau levels $n=2,3,4,5,6$ with $\lambda_{\mathrm{R}}=10 \mathrm{meV}$ and (a) $\lambda_{\mathrm{VZ}}=0$, (b) $\lambda_{\mathrm{VZ}}=2 \mathrm{meV}$, and (c) $\lambda_{\mathrm{VZ}}=4 \mathrm{meV}$.

tures [21]. Rashba SOC combined with valley Zeeman SOC produces particle-hole-symmetric Landau level spectra in both strong- and weak-coupling regimes. This is a consequence of particle-hole-symmetric spectra in the absence of magnetic field [see Figs. 1(b) and 1(c)]. However, as we can see in Fig. 9, the structure of spin splittings and plateaus is more complex in the strong-coupling regime due to the presence of several level crossings, which make it difficult to perform quantitative analysis and estimations based solely on spectra and conductivity profiles. On the other hand, Rashba SOC imprints a clear signature in the fan diagram: for strong $\lambda_{R}$, the fan diagram acquires two extra lateral fans, and the separation between the main fan and the satellite ones is proportional to $\lambda_{R}$. Furthermore, if both $\lambda_{R}$ and $\lambda_{V Z}$ are strong, there is a splitting of the $n=0$ level that does not occur in the case of pure Rashba SOC, as shown in Fig. 9(a).
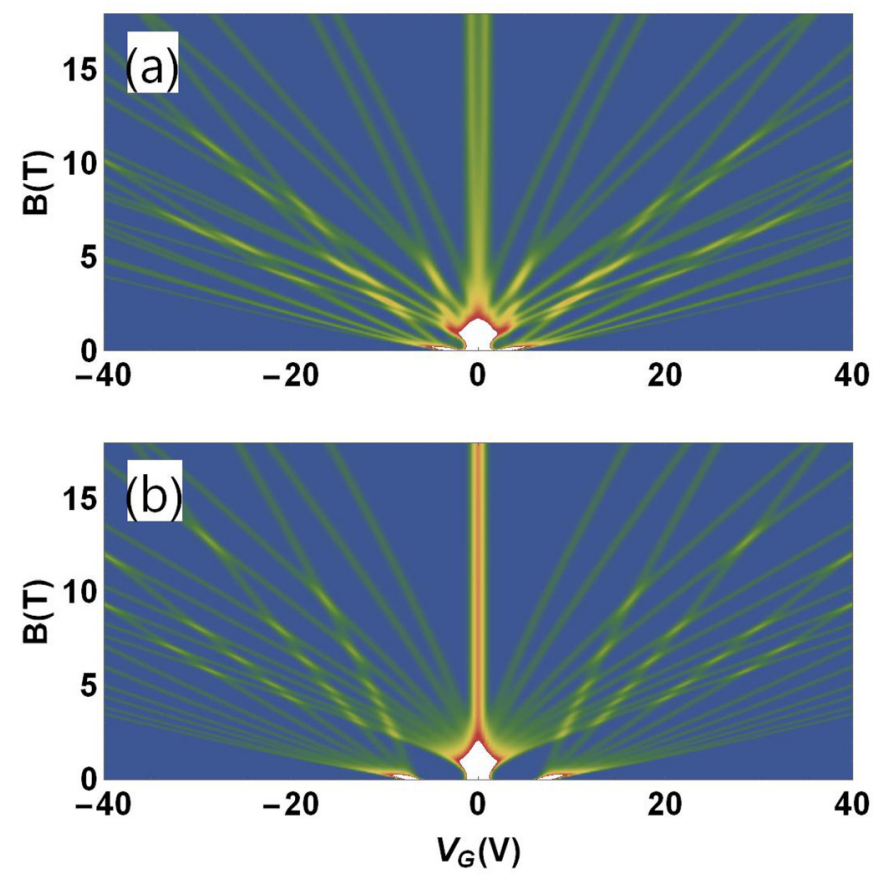

FIG. 9. Landau fan diagrams for (a) $\lambda_{\mathrm{R}}=20 \mathrm{meV}, \lambda_{\mathrm{VZ}}=30 \mathrm{meV}$ and (b) $\lambda_{\mathrm{R}}=40 \mathrm{meV}, \lambda_{\mathrm{VZ}}=20 \mathrm{meV}$.
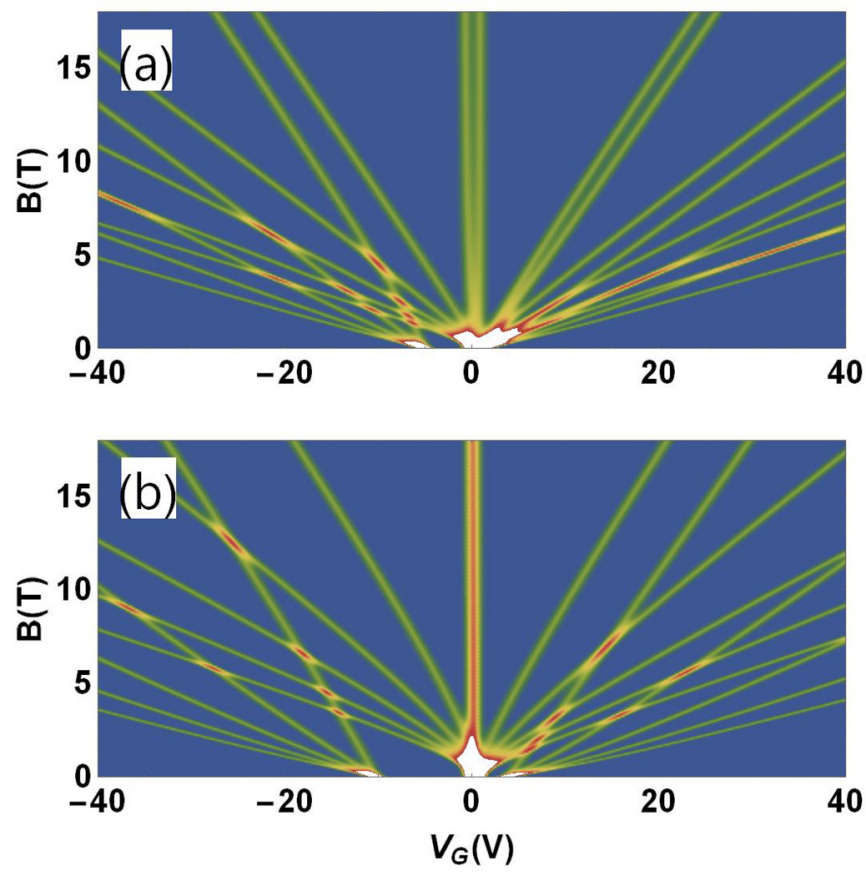

FIG. 10. Landau fan diagram for (a) $\lambda_{\mathrm{R}}=20 \mathrm{meV}, \lambda_{\mathrm{I}}=30 \mathrm{meV}$ and (b) $\lambda_{\mathrm{R}}=40 \mathrm{meV}, \lambda_{\mathrm{I}}=20 \mathrm{meV}$.

\section{Possible applications to other systems}

Instead of considering the interplay between Rashba and valley Zeeman SOCs, we can look at the structure of the Landau fan diagram of graphene with interface-induced Rashba and intrinsic SOCs $\left(\lambda_{R}\right.$ and $\left.\lambda_{I}\right)$, as in the case of graphene intercalated with gold. An example of this intercalated structure is a recent experiment on van der Waals heterostructures of graphene-gold-hBN [23]. We show in Figs. 10(a) and 10(b) the spectra for $\lambda_{I}>\lambda_{R}$ and $\lambda_{I}<\lambda_{R}$, respectively. In both cases, the particle-hole symmetry is broken, a characteristic of the interplay between Kane-Mele SOC and Rashba SOC. For $\lambda_{\mathrm{I}}>\lambda_{\mathrm{R}}$, it presents a topological gap at $V=0$, while the gap is closed if $\lambda_{I}<\lambda_{R}$.

\section{TIGHT-BINDING CALCULATIONS}

\section{A. Graphene-only Hamiltonian for hydrogen on graphene}

Hydrogen adsorption is one of the main sources of contamination when manufacturing graphene. Hydrogen hybridizes with $p_{z}$ orbitals in graphene, modifying the local symmetry from $s p_{2}$ to $s p_{3}$, and creates midgap states [33-36]. To analyze the effect of hydrogenation in the quantum Hall effect, we employ a real-space quantum transport approach [26]. For an optimized use of computational resources, which allow us to study large systems, we need to find a single-orbit tight-binding Hamiltonian for hydrogen on graphene that reproduces a band structure obtained by DFT.

We propose the following graphene-only Hamiltonian for hydrogen adatoms incorporated in a graphene layer:

$$
H_{h}=\varepsilon_{I} c_{I}^{\dagger} c_{I}+t_{I} \sum_{\langle I, j\rangle} c_{I}^{\dagger} c_{j}+\text { H.c. }+\sum_{\langle\langle I, j\rangle\rangle} t_{I}^{(2)} c_{I}^{\dagger} c_{j},
$$



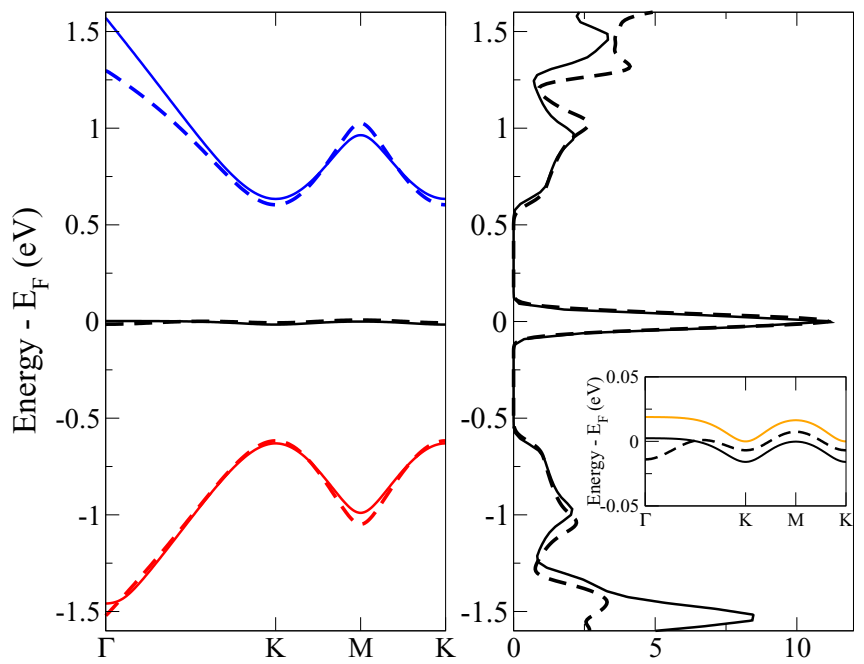

FIG. 11. (a) Band structure showing the conduction (blue), valence (red), and midgap (black) bands and their corresponding (b) density of states obtained through DFT (dashed line) and the fitted TB Hamiltonian (thick line) for a hydrogen adatom on a $5 \times$ 5 graphene supercell. Inset: comparison between our graphene-only model, the DFT result, and the model of Ref. [36] (yellow line) for the midgap band. The parameters of the fit are $t=-2.56 \mathrm{eV}$, $t_{2}=0.010580 \mathrm{eV}, \varepsilon_{I}=-1.5694 \mathrm{eV}, t_{I}=2.6538 \mathrm{eV}$, and $t_{I}^{(2)}=$ $0.29617 \mathrm{eV}$.

where $c_{I}^{\dagger}$ and $c_{I}$ are the creation and annihilation operators for an electron on the adsorption site $I$ and $\langle I, j\rangle$ and $\langle\langle I, j\rangle\rangle$ represent the sum over nearest and next-nearest neighbors of the adsorption site, respectively. $t_{I}$ and $t_{I}^{(2)}$ are parameters that have to be adjusted to properly fit the DFT band structure.

In Fig. 11(a) we show the band structure of a $5 \times 5$ graphene supercell with one hydrogen adatom. The first-principles calculations are based on DFT [37,38] as implemented in the SIESTA code [39], using generalized gradient approximation functional approximation following the Perdew-BurkeErnzerhof approach [40]. The pseudopotential was obtained through the Troullier-Martins scheme [41], and a double- $\zeta$ polarized basis set was used to described the electronic orbitals. The self-consistent cycle was performed using $16 \times 16 \times 1 k$ sampling of the Brillouin zone. The structural relaxation was performed using conjugate gradient minimization until the forces were smaller than $0.01 \mathrm{eV} / \AA$. The gap in the band structure artificially originates from the broken sublattice symmetry due to the arbitrary choice of an adsorption site, which in this case belongs to the sublattice $A$, and disappears when the adatoms are placed randomly because, on average, the symmetry will be restored.

Multiparametric fits such as the present one are usually difficult to perform using deterministic approaches due to the occurrence of a large number of extrema. Therefore, we propose the use of a heuristic algorithm to efficiently perform this task. A simple algorithm that is inspired by natural evolution to find an optimal solution for a given problem is called a genetic algorithm; the scheme is outlined in Fig. 12

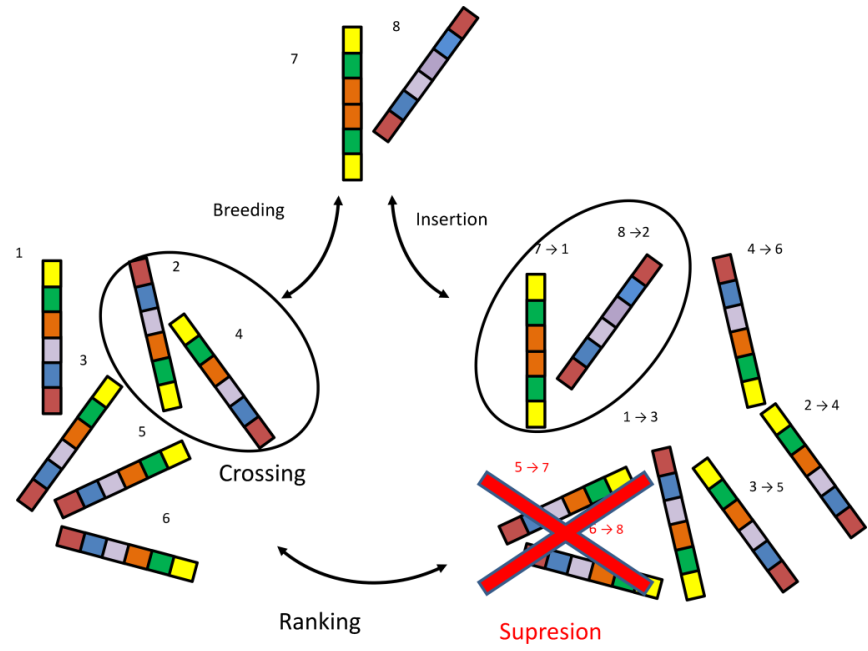

FIG. 12. Schematics of a genetic algorithm.

and observes the following logic:

(1) Primordial generation. A population of $N_{t}$ individuals with random genome vector $\boldsymbol{x}_{p}$ is generated within a hypercube of allowed genomes.

(2) Breeding. $2 N_{p}<N_{t}$ pairs of individuals are chosen randomly and are combined to give rise to $N_{o}<N_{p}$ offspring.

(3) Ranking. All individual parents and offspring are evaluated and ranked through the fitness function.

(4) Reinsertion. The least-fit $N_{o}$ individuals are terminated, keeping the best $N_{t}$ individuals are kept.

(5) Mutation. A stochastic alteration of the $i$ th genome vector occurs with probability $p_{M}$. Then the breeding phase occurs again.

For the primordial generation phase we consider each component of the genome vector to lie within a range of $x \in$ $\left[-x_{0}, x_{0}\right]$, with $x_{0}=1 \mathrm{eV}$. The selection of pairs is performed by following stochastic universal sampling with $N_{p}=N_{t} / 2$, and it is combined using an intermediate recombination

$$
\boldsymbol{x}^{\mathrm{off}}=p \boldsymbol{x}^{\mathrm{p} 1}+(1-p) \boldsymbol{x}^{\mathrm{p} 2},
$$

with $p=-0.25$, producing a set of $N_{o}=N_{t} / 4$ offspring; the fitness function is

$$
f=\left|Y^{\mathrm{DFT}}-Y^{\mathrm{TB}}\right|,
$$

where $Y$ is the band structure shown in Fig. 11(a)

In Fig. 11, we show a comparison between the band structure [Fig. 11(a)] and density of states of our fitted tight-binding Hamiltonian [Fig. 11(b)] and the first-principles results. In the inset of Fig. 11 we also compare our results with a fit that considers an independent orbital for hydrogen [36]. The fit obtained with a genetic algorithm for a graphene-only Hamiltonian is comparable with the one with independent orbitals and has the advantage (if compared with other multiparametric approaches) of being a semiautomatic procedure and using a reduced Hilbert space.

\section{B. Effects of hydrogenation in the quantum Hall effect}

The transport coefficients were computed using a real-space $O(N)$ method based on the Chebyshev expansion of a variant 


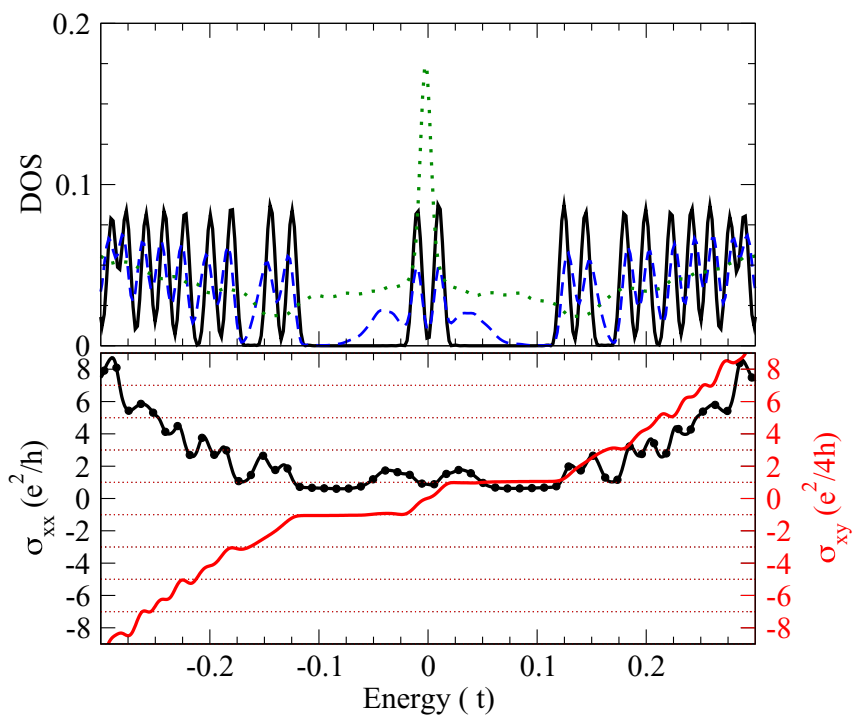

FIG. 13. (a) Density of states for graphene under the action of an external magnetic field of strength $B=13 \mathrm{~T}$, with a proximityinduced SOC defined by the parameters $\lambda_{\mathrm{R}}=20 \mathrm{meV}, \lambda_{a}=-\lambda_{b}=$ $30 \mathrm{meV}$ for different concentrations of hydrogen adatoms: $x_{p}=0$ (dashed blue line), 0.001 (solid black line), and 0.01 (dotted green line). In all cases, there is also a small Anderson disorder with width $W=5 \mathrm{meV}$. (b) Dissipative conductivity (black circles) and Hall conductivity (thick red line) for graphene under the action of an external magnetic field of strength $B=13 \mathrm{~T}$, with a proximityinduced SOC defined by the parameters $\lambda_{\mathrm{R}}=20 \mathrm{meV}$ and $\lambda_{\mathrm{VZ}}=$ $30 \mathrm{meV}$ with $0.1 \%$ hydrogenated impurities

of the Kubo formula, the Kubo-Bastin formula [26],

$$
\begin{aligned}
\sigma_{\alpha \beta}(\mu, T)= & \frac{i \hbar}{\Omega} \int_{-\infty}^{\infty} d \varepsilon f(T, \mu, \varepsilon) \operatorname{Tr}\left\langle j_{\alpha} \delta(\varepsilon-H) j_{\beta} \frac{d G^{+}(H, \varepsilon)}{d \varepsilon}\right. \\
& \left.-j_{\alpha} \frac{d G^{-}(H, \varepsilon)}{d \varepsilon} j_{\beta} \delta(\varepsilon-H)\right\rangle,
\end{aligned}
$$

where $\delta(\varepsilon-H)$ is the $\delta$-function operator, $j_{\alpha}$ is the $\alpha$ component of the current operators, defined as $j_{\alpha} \equiv$ $(1 / i \hbar)\left[x_{\alpha}, H\right], G^{+}(H, \varepsilon)$ and $G^{-}(H, \varepsilon)$ are the advanced and retarded Green's functions, and $f(T, \mu, \varepsilon)$ is the Fermi-Dirac distribution. In this method, the Green's functions and the $\delta$ functions are numerically calculated using the kernel polynomial method [15,26,42-44]. The magnetic field was incorporated by following Peierls's substitution $H_{i, j}=H_{i, j}(B=$ $0) e^{i \phi_{i, j}}$, with $\phi_{i, j}=\int_{\boldsymbol{R}_{i}}^{\boldsymbol{R}_{j}} \boldsymbol{A}(\boldsymbol{r}) \cdot d \boldsymbol{r}$ being the Peierl's phase and $\boldsymbol{A}$ being the vector potential, which was chosen using the Landau gauge $\boldsymbol{A}=\left(B_{0} y, 0,0\right)$

We proceed to analyze the effect of disorder in the QHE in graphene with SOC. Figure 13(a) shows the density of states for $\lambda_{\mathrm{R}}=20 \mathrm{meV}, \lambda_{\mathrm{VZ}}=30 \mathrm{meV}$, which are the same values of the SOC as in Fig. 9(a), for $B=13 \mathrm{~T}$ and different concentrations of hydrogen. The spin-split LL states are still visible with $0.1 \%$ hydrogen, which is the concentration that can be expected from contamination of the samples in experiments. Still, for $1 \%$ hydrogen, the gap at $E=0$ is closed, and the LL spectrum is destroyed. Figure 13(b) presents longitudinal and transverse conductivities for $0.1 \%$ hydrogen. Although not all quantum Hall plateaus are visible in the presence of disorder, the Landau levels, including the spin-split states, are still visible in the longitudinal conductivity. This indicates that our analysis based on the scaling of spin-split states from longitudinal conductivity data should be effective even in the presence of hydrogenation that produces both intravalley and intervalley scattering.

\section{CONCLUSIONS}

Inferring the type and strength of spin-orbit coupling in a graphene heterostructure is a difficult experimental task. Quantum Hall measurements provide a useful tool to understand the physics that govern the charge carriers in two-dimensional materials. The Landau spectrum offers hints about the lowenergy physics of electrons (or holes) and the possible SOCs. Each Landau level in pristine graphene is eightfold degenerate due to spin, pseudospin, and valley isospin quantum numbers. In the presence of SOC, this degeneracy is lifted in a specific way depending on the type of coupling induced in graphene. Motivated by experimental results on magnetotransport in graphene heterostructures, we studied QHE on graphene with different SOCs and provided a simple way to characterize SOCs induced by the proximity effect by analyzing the spin splitting of the Landau levels. By using two different scaling laws, we were able to determine the dominant contribution to the SOC in a graphene layer. Additionally, we used an efficient genetic algorithm strategy together with $a b$ initio calculations to obtain a realistic all-graphene tight-binding Hamiltonian that models hydrogenation in graphene. With this Hamiltonian and a quantum transport approach, we analyzed the effect of hydrogenation on the QHE in graphene with interface-induced SOC. The numerical results indicate that the scaling laws can, in principle, be applied even with $0.1 \%$ hydrogenation.

All results presented here are based on a free-particle approximation. This approximation is appropriate in most situations, but with the evolution of the manufacturing of graphene, cleaner samples have been obtained, and the effects of Coulomb interactions may become important. Due to the Dirac-Weyl nature of electrons on graphene, the role of interactions is different from that in typical materials and is still under intense discussion [45]. The signature of Coulomb interaction for the quantum Hall physics of graphene is the appearance of extra fourfold splittings in the energy spectra, implying intermediate plateaus in the Hall conductivity. For low Landau levels, this splitting has already been observed experimentally with high magnetic fields $[46,47]$. The importance of Coulomb interaction increases exponentially with magnetic field [48], and the symmetry breaking is more pronounced for lower Landau levels, high magnetic fields, and high mobility [49]. The quality of the sample is an important factor to observe Coulomb splitting as disorder decreases the mobility of electrons. Approaches based on a free-particle picture cannot capture the physics of Coulomb interactions, which limits our calculations for samples with moderate electron mobilities and moderate magnetic fields (typically smaller than $15 \mathrm{~T}$ ). The study of the effect of Coulomb interaction on the Landau spectra is beyond the scope of the present work. However, it is important to mention that for experimental transport measurements, the analysis presented here shows that a twofold splitting 
of LLs can be associated with the presence of spin-orbit coupling, while a fourfold splitting is a signature of Coulomb interaction.

\section{ACKNOWLEDGMENTS}

T.G.R. acknowledges the support from the Newton Fund and the Royal Society (UK) through the Newton Advanced Fellowship scheme (Ref. No. NA150043), T.P.C., A.R.R., and T.G.R. thank the Brazilian Agency CNPq for financial support. J.H.G. acknowledges the Severo Ochoa Program (MINECO, Grant No. SEV-2013-0295), the Spanish Ministry of Economy and Competitiveness (Grant No. MAT201233911), the Secretaria de Universidades e Investigacion del Departamento de Economa y Conocimiento de la Generalidad de Catalua, and the European Union Seventh Framework Programme under Grant Agreement No. 604391 Graphene Flagship. This research used computational resources from the Santos Dumont supercomputer at the National Laboratory for Scientific Computing (LNCC/MCTI, Brazil).

\section{APPENDIX: EIGENVALUES AND EIGENSTATES}

The Hamiltonian $H_{\xi}$ is block diagonal, with each block indexed by valley occupation number $n_{\xi}$ (eigenvalue of the number operator $\hat{N}_{\xi}=a_{\xi}^{\dagger} a_{\xi}$ ). The two lowest blocks are $1 \times 1$ and $3 \times 3$ matrices, and higher blocks are $4 \times 4$ matrices for both valleys. The eigenvectors of the first blocks are

$$
\begin{aligned}
& \left|\psi_{0,0}^{+}\right\rangle=|0, B,-\rangle, \\
& \left|\psi_{0,0}^{-}\right\rangle=|0, A,-\rangle,
\end{aligned}
$$

with energies

$$
\begin{aligned}
& E_{0,0}^{+}=\lambda_{b}-\Delta, \\
& E_{0,0}^{-}=\lambda_{a}+\Delta .
\end{aligned}
$$

The first excited block of each valley is given by

$$
H_{1}^{ \pm}=\left(\begin{array}{ccc}
\epsilon_{\alpha}^{ \pm} & 0 & \hbar \omega \\
0 & \epsilon_{\beta}^{ \pm} & \mp 2 i \lambda_{\mathrm{R}} \\
\hbar \omega & \pm 2 i \lambda_{\mathrm{R}} & \epsilon_{\gamma}^{ \pm}
\end{array}\right)
$$

in the basis of $|1, B(A),-\rangle,|0, B(A),+\rangle$, and $|0, A(B),-\rangle$ for $\xi=+(-)$, where $\epsilon_{\alpha}^{ \pm}=\lambda_{b(a)} \mp \Delta, \epsilon_{\beta}^{ \pm}=-\lambda_{b(a)} \mp \Delta$, and $\epsilon_{\gamma}^{ \pm}=-\lambda_{a(b)} \pm \Delta$. The eigenvectors, indexed by $i=1,2,3$, are given by

$$
\begin{aligned}
\left|\psi_{1, i}^{ \pm}\right\rangle= & \alpha_{1, i}^{ \pm}|1, B(A),-\rangle+\beta_{1, i}^{ \pm}|0, B(A),+\rangle \\
& +\gamma_{1, i}^{ \pm}|0, A(B),-\rangle
\end{aligned}
$$

with coefficients

$$
\begin{gathered}
\alpha_{1, i}^{ \pm}=\left[\left(2 \lambda_{\mathrm{R}}\right)^{2}-\left(\epsilon_{\beta}^{ \pm}-E_{1, i}^{ \pm}\right)\left(\epsilon_{\gamma}^{ \pm}-E_{1, i}^{ \pm}\right)\right] / \sqrt{D_{ \pm}}, \\
\beta_{1, i}^{ \pm}= \pm i\left(2 \lambda_{\mathrm{R}}\right)(\hbar \omega) / \sqrt{D_{ \pm}} \\
\gamma_{1, i}^{ \pm}=(\hbar \omega)\left(\epsilon_{\beta}^{ \pm}-E_{1, i}^{ \pm}\right) / \sqrt{D_{ \pm}}
\end{gathered}
$$

where $\quad D_{ \pm}=(\hbar \omega)^{2}\left(\epsilon_{\beta}^{ \pm}-E_{1, i}^{ \pm}\right)^{2}+\left[\left(2 \lambda_{\mathrm{R}}\right)^{2}-\left(\epsilon_{\beta}^{ \pm}-\right.\right.$ $\left.\left.E_{1, i}^{ \pm}\right)\left(\epsilon_{\gamma}^{ \pm}-E_{1, i}^{ \pm}\right)\right]^{2}+(\hbar \omega)^{2}\left(2 \lambda_{\mathrm{R}}\right)^{2}$.

Finally, for $n_{\xi} \geqslant 2$ the blocks, written in the basis $\left|n_{ \pm}-2, A(B),+\right\rangle,\left|n_{ \pm}, B(A),-\right\rangle,\left|n_{ \pm}-1, B(A),+\right\rangle$, and $\left|n_{ \pm}-1, A(B),-\right\rangle$, are given by

$H_{n}^{ \pm}=\left(\begin{array}{cccc}E_{a}^{ \pm} & 0 & \hbar \omega \sqrt{n_{ \pm}-1} & 0 \\ 0 & E_{b}^{ \pm} & 0 & \hbar \omega \sqrt{n_{ \pm}} \\ \hbar \omega \sqrt{n_{ \pm}-1} & 0 & E_{c}^{ \pm} & \mp 2 i \lambda_{\mathrm{R}} \\ 0 & \hbar \omega \sqrt{n_{ \pm}} & \pm 2 i \lambda_{\mathrm{R}} & E_{d}^{ \pm}\end{array}\right)$,

where $E_{a}^{ \pm}=\lambda_{a(b)} \pm \Delta, E_{b}^{ \pm}=\lambda_{b(a)} \mp \Delta, E_{c}^{ \pm}=-\lambda_{b(a)} \mp \Delta$, and $E_{d}^{ \pm}=-\lambda_{a(b)} \pm \Delta$. The eigenstates are given by

$$
\begin{aligned}
\left|\psi_{n, i}^{ \pm}\right\rangle= & a_{n, i}^{ \pm}\left|n_{ \pm}-2, A(B),+\right\rangle+b_{n, i}^{ \pm}\left|n_{ \pm}, B(A),-\right\rangle \\
& +c_{n, i}^{ \pm}\left|n_{ \pm}-1, B(A),+\right\rangle+d_{n, i}^{ \pm}\left|n_{ \pm}-1, A(B),-\right\rangle,
\end{aligned}
$$

and their coefficients can be written in terms of four eigenvalues of each block $E_{n, i}^{ \pm}$:

$$
\begin{aligned}
b_{n, i}^{ \pm}= & {\left[1+\frac{\left(E_{b}^{ \pm}-E_{n, i}^{ \pm}\right)^{2}}{n_{ \pm}(\hbar \omega)^{2}}+n_{ \pm}\left(\frac{\hbar \omega}{2 \lambda_{\mathrm{R}}}\right)^{2}\right.} \\
& \times\left(1+\frac{(\hbar \omega)^{2}\left(n_{ \pm}-1\right)}{\left(E_{a}^{ \pm}-E_{n, i}^{ \pm}\right)^{2}}\right) \\
& \left.\times\left(1-\frac{\left(E_{b}^{ \pm}-E_{n, i}^{ \pm}\right)\left(E_{d}^{ \pm}-E_{n, i}^{ \pm}\right)}{(\hbar \omega)^{2} n_{ \pm}}\right)^{2}\right]^{-1 / 2}, \\
a_{n, i}^{ \pm}= & -\frac{i(\hbar \omega)^{2} \sqrt{n_{ \pm}\left(n_{ \pm}-1\right)}}{\left( \pm 2 \lambda_{\mathrm{R}}\right)\left(E_{a}^{ \pm}-E_{n, i}^{ \pm}\right)} \\
& \times\left(1-\frac{\left(E_{b}^{ \pm}-E_{n, i}^{ \pm}\right)\left(E_{d}^{ \pm}-E_{n, i}^{ \pm}\right)}{(\hbar \omega)^{2} n_{ \pm}}\right) b_{n, i}^{ \pm}, \quad(\mathrm{A} \\
c_{n, i}^{ \pm}= & \frac{i \hbar \omega \sqrt{n_{ \pm}}}{\left( \pm 2 \lambda_{\mathrm{R}}\right)}\left(1-\frac{\left(E_{b}^{ \pm}-E_{n, i}^{ \pm}\right)\left(E_{d}^{ \pm}-E_{n, i}^{ \pm}\right)}{(\hbar \omega)^{2} n_{ \pm}}\right) b_{n, i}^{ \pm},
\end{aligned}
$$

$$
d_{n, i}^{ \pm}=-\frac{\left(E_{b}^{ \pm}-E_{n, i}^{ \pm}\right)}{\hbar \omega \sqrt{n_{ \pm}}} b_{n, i}^{ \pm}
$$

[1] M. Gmitra, D. Kochan, P. Högl, and J. Fabian, Phys. Rev. B 93, 155104 (2016).

[2] M. Venkata Kamalakar, A. Dankert, J. Bergsten, T. Ive, and S. P. Dash, Appl. Phys. Lett. 105, 212405 (2014).
[3] P. J. Zomer, M. H. D. Guimarães, N. Tombros, and B. J. van Wees, Phys. Rev. B 86, 161416 (2012).

[4] C. R. Dean, L. Wang, P. Maher, C. Forsythe, F. Ghahari, Y. Gao, J. Katoch, M. Ishigami, P. Moon, M. Koshino, T. Taniguchi, K. 
Watanabe, K. L. Shepard, J. Hone, and P. Kim, Nature (London) 497, 598 (2013).

[5] B. Hunt, J. D. Sanchez-Yamagishi, A. F. Young, M. Yankowitz, B. J. LeRoy, K. Watanabe, T. Taniguchi, P. Moon, M. Koshino, P. Jarillo-Herrero, and R. C. Ashoori, Science 340, 1427 (2013).

[6] W. Han, R. K. Kawakami, M. Gmitra, and J. Fabian, Nat. Nanotechnol. 9, 794 (2014).

[7] A. K. Geim and I. V. Grigorieva, Nature (London) 499, 419 (2013).

[8] S. Konschuh, M. Gmitra, and J. Fabian, Phys. Rev. B 82, 245412 (2010).

[9] H. Min, J. E. Hill, N. A. Sinitsyn, B. R. Sahu, L. Kleinman, and A. H. MacDonald, Phys. Rev. B 74, 165310 (2006).

[10] Y. Yao, F. Ye, X.-L. Qi, S.-C. Zhang, and Z. Fang, Phys. Rev. B 75, 041401(R) (2007).

[11] A. H. Castro Neto and F. Guinea, Phys. Rev. Lett. 103, 026804 (2009).

[12] A. Ferreira, T. G. Rappoport, M. A. Cazalilla, and A. H. Castro Neto, Phys. Rev. Lett. 112, 066601 (2014).

[13] H.-Y. Yang, C. Huang, H. Ochoa, and M. A. Cazalilla, Phys. Rev. B 93, 085418 (2016).

[14] M. Milletari and A. Ferreira, Phys. Rev. B 94, 134202 (2016).

[15] J. H. Garcia and T. G. Rappoport, 2D Mater. 3, 024007 (2016).

[16] J. H. G. Aguilar, A. W. Cummings, and S. Roche, Nano Lett. 17, 5078 (2017).

[17] Z. Wang, C. Tang, R. Sachs, Y. Barlas, and J. Shi, Phys. Rev. Lett. 114, 016603 (2015).

[18] J. B. S. Mendes, O. A. Santos, L. M. Meireles, R. G. Lacerda, L. H. Vilela-Leao, F. L. A. Machado, R. L. Rodriguez-Suarez, A. Azevedo, and S. M. Rezende, Phys. Rev. Lett. 115, 226601 (2015).

[19] A. Avsar, J. Y. Tan, T. Taychatanapat, J. Balakrishnan, G. K. W. Koon, Y. Yeo, J. Lahiri, A. Carvalho, A. S. Rodin, E. C. T. O'Farrell, G. Eda, A. H. C. Neto, and B. Ozyilmaz, Nat. Commun. 5, 4875 (2014).

[20] Z. Wang, D.-K. Ki, H. Chen, H. Berger, A. H. MacDonald, and A. F. Morpurgo, Nat. Commun. 6, 8339 (2015).

[21] Z. Wang, D.-K. Ki, J. Y. Khoo, D. Mauro, H. Berger, L. S. Levitov, and A. F. Morpurgo, Phys. Rev. X 6, 041020 (2016).

[22] B. Yang, M.-F. Tu, J. Kim, Y. Wu, H. Wang, J. Alicea, R. Wu, M. Bockrath, and J. Shi, 2D Mater. 3, 031012 (2016).

[23] E. C. T. O'Farrell, J. Y. Tan, Y. Yeo, G. K. W. Koon, B. Ozyilmaz, K. Watanabe, and T. Taniguchi, Phys. Rev. Lett. 117, 076603 (2016).

[24] Y. K. Lou, J. Xu, T. Zhu, G. Wu, E. J. McCormick, W. Zhan, M. R. Neupane, and R. K. Kawakami, Nano Lett. 17, 3877 (2017).
[25] A. Avsar, D. Unuchek, J. Liu, O. Lopez Sanchez, K. Watanabe, T. Taniguchi, B. Özyilmaz, and A. Kis, ACS Nano 11, 11678 (2017).

[26] J. H. Garcia, L. Covaci, and T. G. Rappoport, Phys. Rev. Lett. 114, 116602 (2015).

[27] Y. A. Bychkov and E. I. Rashba, J. Phys. C 17, 6039 (1984).

[28] E. I. Rashba, Phys. Rev. B 79, 161409(R) (2009).

[29] C. L. Kane and E. J. Mele, Phys. Rev. Lett. 95, 226801 (2005).

[30] C. L. Kane and E. J. Mele, Phys. Rev. Lett. 95, 146802 (2005).

[31] G. D. Mahan, Many-Particle Physics, 3rd ed. (Plenum, New York, 2010).

[32] N. M. R. Peres, Rev. Mod. Phys. 82, 2673 (2010).

[33] V. M. Pereira, J. M. B. Lopes dos Santos, and A. H. Castro Neto, Phys. Rev. B 77, 115109 (2008).

[34] J. Bang and K. J. Chang, Phys. Rev. B 81, 193412 (2010).

[35] S. Ihnatsenka and G. Kirczenow, Phys. Rev. B 83, 245442 (2011).

[36] M. Gmitra, D. Kochan, and J. Fabian, Phys. Rev. Lett. 110, 246602 (2013).

[37] P. Hohenberg and W. Kohn, Phys. Rev. 136, B864 (1964).

[38] W. Kohn and L. J. Sham, Phys. Rev. 140, A1133 (1965).

[39] J. M. Soler, E. Artacho, J. D. Gale, A. García, J. Junquera, P. Ordejón, and D. Sánchez-Portal, J. Phys. Condens. Matter 14, 2745 (2002).

[40] J. P. Perdew, K. Burke, and M. Ernzerhof, Phys. Rev. Lett. 77, 3865 (1996).

[41] N. Troullier and J. L. Martins, Phys. Rev. B 43, 1993 (1991).

[42] A. Weisse, G. Wellein, A. Alvermann, and H. Fehske, Rev. Mod. Phys. 78, 275 (2016).

[43] L. Covaci, F. M. Peeters, and M. Berciu, Phys. Rev. Lett. 105, 167006 (2010).

[44] R. Silver and H. Röder, Int. J. Mod. Phys. C 5, 735 (1994).

[45] V. N. Kotov, B. Uchoa, V. M. Pereira, F. Guinea, and A. H. Castro Neto, Rev. Mod. Phys. 84, 1067 (2012).

[46] Y. Zhang, Z. Jiang, J. P. Small, M. S. Purewal, Y.-W. Tan, M. Fazlollahi, J. D. Chudow, J. A. Jaszczak, H. L. Stormer, and P. Kim, Phys. Rev. Lett. 96, 136806 (2006).

[47] A. F. Young, C. R. Dean, L. Wang, H. Ren, P. Cadden-Zimansky, K. Watanabe, T. Taniguchi, J. Hone, K. L. Shepard, and P. Kim, Nat. Phys. 8, 550 (2012).

[48] M. O. Goerbig, Rev. Mod. Phys. 83, 1193 (2011).

[49] K. Nomura and A. H. MacDonald, Phys. Rev. Lett. 96, 256602 (2006). 